\title{
Sri Lanka : un pays qui s'enfonce de nouveau dans la guerre
}

Delon Madavan

\section{OpenEdition}

\section{Journals}

\section{Édition électronique}

URL : http://journals.openedition.org/echogeo/2543

DOI : 10.4000/echogeo.2543

ISSN : 1963-1197

\section{Éditeur}

Pôle de recherche pour l'organisation et la diffusion de l'information géographique (CNRS UMR 8586)

\section{Référence électronique}

Delon Madavan, «Sri Lanka : un pays qui s'enfonce de nouveau dans la guerre », EchoGéo [En ligne], Sur le Vif, mis en ligne le 08 avril 2008, consulté le 30 avril 2019. URL : http://journals.openedition.org/ echogeo/2543; DOl : 10.4000/echogeo.2543

Ce document a été généré automatiquement le 30 avril 2019.

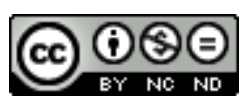

EchoGéo est mis à disposition selon les termes de la licence Creative Commons Attribution - Pas d'Utilisation Commerciale - Pas de Modification 4.0 International 


\title{
Sri Lanka: un pays qui s'enfonce de nouveau dans la guerre
}

\author{
Delon Madavan
}

1 Le soixantième anniversaire de l'indépendance de l'île a été fêté dans une atmosphère très lourde. En effet, les espoirs de paix suscités par l'accord de cessez-le-feu, conclu entre le gouvernement de Sri Lanka et le mouvement séparatiste du Liberation Tigers of Tamil Eelam (LTTE ${ }^{1}$ ) en février 2002, font désormais partie du passé. Déjà en 2006, la reprise de combats intenses à l'Est et au Nord du pays, l'assassinat de politiciens, de personnes issues de la société civile ou des seize membres de l'ONG Action Contre la Faim faisaient de ce pays un Etat en guerre non déclarée. Le retrait unilatéral du Président Rajapakse de l'accord de cessez-le-feu en janvier, les opérations militaires dans le Nord et l'Est, et les attentats suicides dans la capitale et le Sud, ont fait basculer Sri Lanka dans la guerre. Une fois de plus, la dégradation de la situation politique risque de mettre à mal l'unité nationale et d'exacerber au contraire la montée de l'intégrisme et du repli communautaire. Comment peut-on expliquer la détérioration de la situation politique et quelles en sont les différentes conséquences?

2 Nous verrons que les positions des principaux protagonistes ne pouvaient que conduire à la dégradation de la situation politique. Puis nous examinerons les conséquences géopolitiques induites par ce retour à la guerre et ses impacts pour la population civile. 


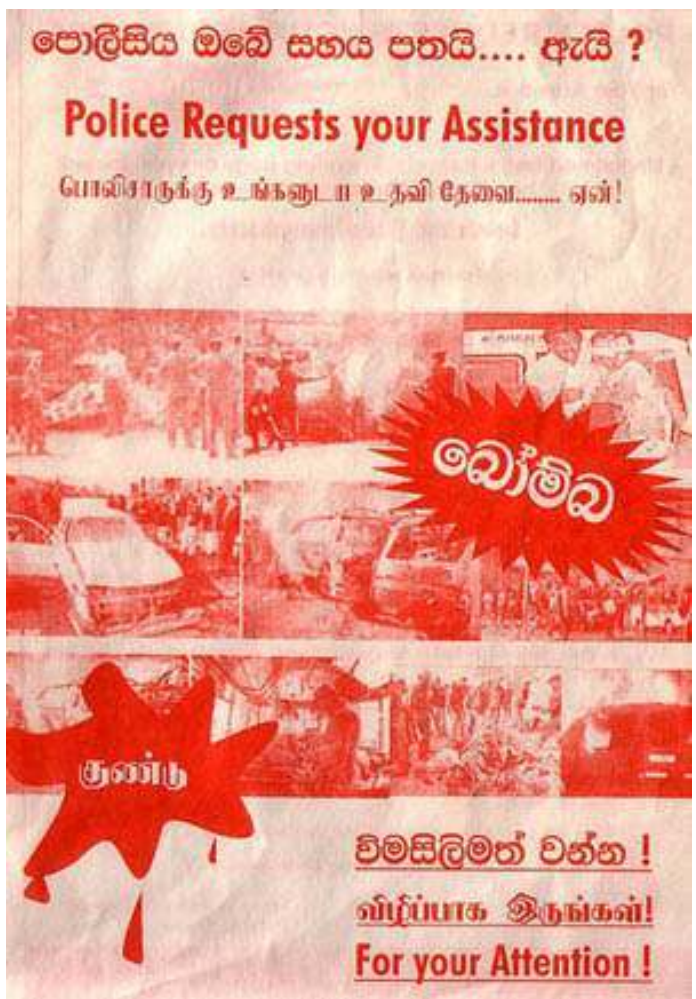

Les principaux acteurs chargés de s'entendre pour résoudre le conflit (M. Prebhakaran, leader du LTTE, et le Président de Sri Lanka, M. Rajapakse) sont finalement les premiers à vouloir mettre un terme au statut quo et à imposer par les armes leurs aspirations pour le pays.

L'actuel Président Rajapakse a été élu de peu en novembre 2005 après avoir fait campagne en promettant de vaincre le LTTE et de mettre fin au rôle de médiateur de la Norvège. Son adversaire Wickremesinghe, alors Premier Ministre, défendait, lui, une solution négociée pour mettre un terme à un conflit qui s'éternisait. Le nouvel homme fort de Colombo n'était ainsi prêt à faire aucune concession aux Tigres (surnom donné aux militants du LTTE). Pour gouverner, le Président Rajapakse a dû s'allier au Parlement avec deux partis politiques ultranationalistes cinghalais : le Janatha Vimukthi Peramuna (JVP²) et le Jathika Hela Urumaya $\left(\mathrm{JHU}^{3}\right)$. Ces partenaires du parti au pouvoir, le Sri Lanka Freedom Parti (SLFP), ont fait pression sur le gouvernement en décembre 2007, en acceptant de ne voter le budget qu'à la troisième lecture en échange du retrait de l'accord de cessez-le-feu.

La stratégie du gouvernement central est indéniablement d'anéantir militairement le LTTE pour résoudre le conflit. Pour cela, les dirigeants projettent de reconquérir l'ensemble des territoires sous le contrôle du LTTE au Nord (Wanni) et à l'Est, et si possible de déstabiliser le mouvement rebelle en essayant de capturer, voire d'assassiner le chef historique des Tigres.

6 Malgré le discours officiel du LTTE, qui se dit favorable à une résolution du conflit par l'adoption d'un régime fédéral avec une très large autonomie pour les provinces historiquement à majorité tamoule, l'objectif ultime des Tigres reste la création de l'Eelam, un Etat indépendant pour les Tamouls. C'est pourquoi le LTTE a favorisé l'élection de Rajapakse, au détriment de Wickremesinghe qui prônait la réconciliation 
nationale et l'adoption d'un système fédéral, en boycottant les élections présidentielles dans les régions de la province Nord alors sous son contrôle. A Jaffna, tenue par les forces gouvernementales, les Tigres ont intimidé les civils en lançant des grenades dans des bureaux de vote. Ainsi, de nombreux Jaffnais, qui auraient voté pour le candidat de la réconciliation, ont préféré s'abstenir. Cela a bénéficié à Rajapakse qui disposait de peu de réserve de voix dans ces territoires tamouls. Pour les Tigres, Wickremesinghe était plus dangereux car il offrait aux Tamouls, las de la guerre, une alternative crédible. Il aurait ainsi pu mettre à mal la légitimité du LTTE d'être le seul représentant des Tamouls, et son élection aurait pu porter un coup fatal à l'espoir de voir naître un jour l'Eelam. A l'inverse, l'élection de Rajapakse à la tête de l'Etat sri lankais leur assurait d'avoir un intermédiaire avec qui ils étaient sûrs de ne pas pouvoir s'entendre, sans que l'on puisse leur reprocher d'être responsables de l'échec des négociations.

Le retrait du gouvernement de l'accord de cessez-le-feu s'est suivi d'une escalade de la violence. En effet, la qualification du LTTE de groupe terroriste par de nombreux pays, le retrait des observateurs internationaux et la quasi-absence de journalistes étrangers ont laissé les mains libres au gouvernement pour lancer des opérations militaires de grande ampleur sans qu'il n'y ait de témoin pour freiner son action. Ainsi, les bombardements et les affrontements se sont multipliés dans les provinces Nord et Est. La propagande dans les journaux rapporte les victoires irrésistibles de l'armée nationale et la chute annoncée du fief des Tigres, Kilinochchi. Le gouvernement s'efforce d'alimenter l'idée qu'une victoire totale est possible et qu'il contrôle la situation, alors que sur le terrain ce constat semble loin d'être évident.

Le LTTE est un mouvement aguerri par 25 ans de guerre et il est peu vraisemblable que les forces régulières réussissent si facilement à remporter une victoire totale. En effet, les Tigres semblent capables de soutenir une guerre conventionnelle au Nord, non conventionnelle dans l'Est, et de commettre des attentats suicides ou de poser des bombes dans la capitale et le Sud de l'île. Par ailleurs, même si la perte des possessions contrôlées par le LTTE serait symboliquement terrible pour lui, c'est un mouvement qui a déjà fait ses preuves contre les armées sri lankaise et indienne, malgré son infériorité numérique et matérielle, grâce à une lutte de guérilla. Il semble donc difficile de venir totalement à bout des Tigres par les armes. Au contraire, les Tigres risquent d'attaquer des cibles stratégiques aussi bien économiques (aéroport Katunayake, port de Trincomalee) que politiques (parlementaires, ministres, voire le président lui-même). La tactique du gouvernement est très critiquable car même s'il réussit un jour à venir à bout du LTTE, cela se sera soldé par d'innombrables pertes humaines.

Dans ce contexte, les défenseurs d'une solution politique pour résoudre le conflit, la liberté d'expression et les droits de l'Homme sont de nouveau menacés. Les membres de la société civile et les chercheurs sont pourchassés et stigmatisés comme "traitres à la patrie » par les partisans de la solution militaire pour régler le conflit. Les médias indépendants, qu'ils soient pro-tamouls ou qu'ils contestent la politique gouvernementale, sont interdits ou très contrôlés. La marge de manœuvre des pacifistes est plus que réduite actuellement.

La population civile est de nouveau victime de l'irresponsabilité des belligérants. Dans toutes les parties de l'île, les populations civiles et les Tamouls en particulier souffrent du retour de la guerre.

11 Jaffna, qui avait été l'un des principaux théâtres de la guerre depuis 1987, a profité de l'accord de cessez-le-feu pour se reconstruire et certains habitants commençaient à peine 
à se réinstaller. Depuis la dégradation de la situation politique, les Jaffnais connaissent de nouveaux les couvre-feux et les pénuries de nourriture, de médicaments et de pétrole. Le risque de redevenir l'un des principaux objectifs à conquérir pour les belligérants met à mal la renaissance de la ville. Son développement économique est déjà enrayé depuis que la route $\mathrm{A} 9$, qui relie la ville à la capitale, a été fermée. De nombreux Jaffnais ont préféré quitter leur région par précaution pour gagner la capitale.

Dans les territoires contrôlés par le LTTE, comme Wanni, la population, dont la mobilité vers le reste du pays est déjà très limitée par les séparatistes tamouls, vit dans des conditions matérielles très sommaires et se retrouve au premier front. En effet, l'armée gouvernementale a déjà commencé à bombarder les positions des Tigres dans cette partie de l'île. De plus, pour faire face à l'intensification des affrontements armés, le LTTE vient d'exiger que chaque famille vivant dans le territoire des Tigres envoie deux enfants, un garçon et une fille, dans les rangs du groupe paramilitaire. Les civils se retrouvent ainsi malgré eux embrigadés dans le conflit.

Dans la capitale, la multiplication des attentats renforce le sentiment d'incertitude et de méfiance vis-à-vis des Tamouls originaires du Nord ou de l'Est. Les Tamouls ayant réussi à quitter leur province d'origine préfèrent souvent s'installer dans des quartiers de Colombo, comme Wellawatte ou Dehiwalla, où habitent déjà de nombreux membres de leur communauté ethnique. La minorité tamoule doit faire face à de nombreux problèmes. Ainsi, ils doivent s'inscrire au commissariat le plus proche. En effet, les Tamouls doivent être capables de fournir leur carte d'identité et leur autorisation de résidence à Colombo lors des contrôles routiers ou d'identité. Ceux qui ne sont pas capables de fournir ces documents risquent d'être arrêtés pour vérification et détenus cinq jours. Les Tamouls, en particulier ceux originaires des provinces Nord ou Est, limitent leurs déplacements pour éviter tout problème, notamment le soir après $20 \mathrm{~h}$. D'ailleurs lorsqu'ils sont obligés de se déplacer la nuit, ils préfèrent prendre le bus plutôt que les three wheels qui sont contrôlés.

La liberté de circulation est ainsi très limitée et l'assassinat d'un parlementaire tamoul du parti de l'opposition, T. Maheswaran, dans un temple hindouiste a poussé certains Tamouls à les éviter aussi. L'horizon des Tamouls se réduit souvent aux lieux qu'ils sont obligés de fréquenter, c'est-à-dire leur maison, leur lieu de travail et l'école des enfants. Leur cercle de sociabilité reste restreint et ils préfèrent autant que possible rester chez eux ou chez l'un de leurs proches. Mais même sous leur toit, les Tamouls ne se sentent plus en sécurité : les perquisitions nocturnes dans les quartiers réputés pour abriter une importante communauté tamoule font souvent l'objet de contrôles afin de vérifier qu'il n'y a pas dans ces lieux des membres ou des sympathisants de LTTE. Ce harcèlement dû à l'ethnicité donne le sentiment aux Tamouls d'être vulnérables et en danger.

15 Tout ceci renforce la volonté des Tamouls de quitter leur pays. Au final, la reprise du conflit devient paradoxalement une opportunité aux candidats à l'exil, car elle augmente leurs chances d'être accueillis comme réfugiés politiques, si toutefois ils réussissent à atteindre un pays occidental.

16 Aucune solution durable n'est proposée par le gouvernement, sauf la guerre. Les deux principaux protagonistes du conflit à Sri Lanka se disent prêts adopter un régime fédéral mais dans une version épurée pour Rajapakse et uniquement dans le discours pour le LTTE, dont les aspirations demeurent la création de l'Eelam tamoul. Cela explique que l'île sombre une nouvelle fois dans la guerre. Le gouvernement et le LTTE n'ont tiré aucune leçon des erreurs du passé. En effet, ils reproduisent le scénario de 1987, lorsque le 
Président de l'époque avait préféré la solution militaire pour en finir avec le problème des Tigres, sans en considérer les répercussions sur les populations civiles. La reprise des affrontements se traduit par un repli communautaire et retarde l'espoir de voir cohabiter pacifiquement Cingalais, Tamouls et Musulmans. Au contraire, ce nouveau coup porté à l'harmonie intercommunautaire à Sri Lanka renforce la volonté des Tamouls de gagner l'étranger et de rejoindre une diaspora qui n'a cessé de croître depuis le début des tensions intercommunautaires dans l'île.

\section{NOTES}

1. LTTE (Liberation Tigers of Tamil Eelam) : est un mouvement indépendantiste, fondé en 1976 par V. Prebhakaran, dont le but affiché est de défendre les Tamouls de Sri Lanka. Pour cela, l'organisation demande le droit à l'autodétermination et la création d'un Etat, le Tamil Eelam, dans le nord et l'est de l'île. Pour obtenir leur indépendance, les Tigres se sont engagés dans une lutte armée avec le gouvernement central. Ce mouvement indépendantiste n'hésite pas à utiliser la force pour éliminer ses rivaux politiques afin de s'affirmer comme le seul représentant les intérêts des Tamouls de l'île. Les Tigres utilisent aussi des attentats suicide comme moyen d'action pour faire avancer leur cause (Rajiv Gandhi, Premadasa, etc.). Le LTTE s'est affirmé depuis 1987 comme l'acteur incontournable de la scène politique nationale. En effet, le mouvement a réussi à faire face aux différents assauts de l'armée gouvernementale et même aux forces indiennes qui ont tenté un temps de lui faire déposer les armes par la force. Les Tigres dirigent comme un Etat souverain des territoires dans le nord et l'est du pays qu'ils contrôlent militairement. Bien que le LTTE soit présenté comme le mouvement séparatiste le plus puissant et organisé du monde, il ne dispose pas du soutien de la communauté internationale. En effet, plus de trente-deux pays (dont l'Inde, les Etats-Unis et l'Union Européenne) le considèrent comme une organisation terroriste.

2. JVP (Janatha Vimikhti Peramuna ou Front de Liberation du Peuple) : est un mouvement révolutionnaire marxiste lancé dans les années 1960 par des admirateurs de Guevara afin de renverser le pouvoir en place. Ce parti a été interdit à plusieurs reprises à cause des actions violentes qu'il a lancé pour déstabiliser les gouvernements en place (insurrections 1971 et 1987-89, assassinats politiques, attaques des forces de sécurités) et a été la cible de féroce répression. Après l'échec de la rébellion qu'il a lancé en 1971 dans le Sud de l'île, le parti a évolué dans un sens ultranationaliste. Le parti veut protéger le pays de l'expansionnisme indien ou de l'influence de tout pays tiers. A ce titre, le JVP est très hostiles aux Tamouls des plantations qu'il considère comme étant des éléments de cette expansionnisme. Le parti exploite également le sentiment anti tamoul en se présentant comme la force politique la plus hostile à la création d'un Etat tamoul indépendant. Ainsi, le JVP, qui a été de nouveau autorisé depuis 1994, s'oppose à tout accord de Paix avec le LTTE. Il souhaite au contraire affaiblir militairement le mouvement rebelle tamoul et ne discuter avec ce dernier que s'il rend les armes, renonce à la demande d'un Etat indépendant. Depuis 2001, le JVP est rentré dans la coalition des partis au pouvoir 
3. JHU (Jathika Hela Urumaya ou le Parti de l'Héritage National): lancé en 2004, ce parti dirigé par des moines bouddhistes se présente comme garant des valeurs traditionnelles du pays. Le JHU exalte le nationalisme cingalais et veut défendre la place du bouddhisme dans la société sri lankaise. Le parti s'est allié avec le JVP pour s'opposer aux plans de Paix. Ces moines politisés défendent la position de se débarrasser du problème terroriste par la force et militent pour le maintien d'un Etat centralisé à Sri Lanka.

\section{RÉSUMÉS}

Le retrait unilatéral de l'accord de cessez-le-feu par le Président Rajapakse marque le retour officiel à l'état de guerre à Sri Lanka. L'île est meurtrie par les affrontements opposant l'armée gouvernementale aux militants séparatistes tamouls. Les civils se retrouvent encore prisonniers du cynisme des belligérants, qui préfèrent ignorer les répercussions de leurs actes pour arriver à leur fin, quitte à mettre en danger l'harmonie intercommunautaire dans l'île.

President Rajapakse's decision to withdraw from the cease-fire agreement marks the official return to state of war in Sri Lanka. The island has immensely suffered by clashes between government forces and tamil separatist militants. Once again, the civilians are prisoners of cynicism of belligerents, those prefer ignoring the consequences of their acts to archieve their goal, even if it means endangering intercultural harmony in the island.

\section{INDEX}

Mots-clés : Sri Lanka, guerre, Cingalais, Tamoul, LTTE, Rajapakse

Keywords : war, sinhalese, tamil

\section{AUTEUR}

\section{DELON MADAVAN}

Delon Madavan est doctorant et moniteur en géographie à l'Université Paris-Sorbonne. Il travaille actuellement, sous la direction de Monsieur Olivier Sevin, sur la minorité tamoule à Colombo, Kuala Lumpur et Singapour. D. Madavan a publié dans la collection Grafigeo de l'UMR PRODIG : Jaffna et le conflit intercommunautaire à Sri Lanka. Il est également l'auteur des articles sur Colombo et la géographie du Sri Lanka de la dernière édition de l'Encyclopédie Universalis. 